\title{
Structure and Properties of Soft-Magnetic Amorphous Bilayer Ribbons
}

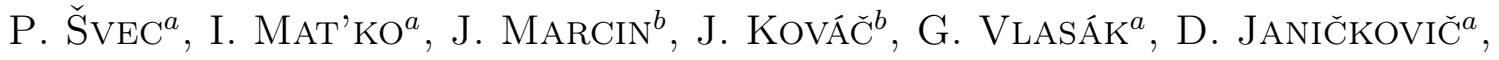 \\ I. ŠKORVÁNEK ${ }^{b}$ AND P. ŠVEC SR. ${ }^{a}$ \\ ${ }^{a}$ Institute of Physics, Slovak Academy of Sciences, Dúbravská cesta 9, 84511 Bratislava, Slovakia
} ${ }^{b}$ Institute of Experimental Physics, Slovak Academy of Sciences, Watsonova 47, 04001 Košice, Slovakia

Rapidly quenched bilayers consisting of $\mathrm{Fe}-\mathrm{Si}-\mathrm{B}$ and $\mathrm{Co}-\mathrm{Si}-\mathrm{B}$ layers have been prepared by planar flow casting from a single crucible with two nozzles. Temperature dependences of electrical resistivity, dilatation and magnetization have been investigated in the amorphous state and during crystallization of both layers. Preliminary studies of the structure of the layers and of the interlayer have been performed by X-ray diffraction, cross-sectional electron microscopy and microanalysis. From the results it seems evident that the process of connection of the two layers during preparation takes place by solidification with only a small extent of mutual interdiffusion of component atoms localized to a narrow well-defined interface, leading to mechanically solid connection between the two layers. The effect of combined presence of two different soft magnetic alloys on the overall magnetic properties is discussed with respect to potential applications of such materials.

PACS numbers: 68.37.Hk, 68.37.Lp

\section{Introduction}

The preparation of bilayers or multilayers for technological applications (bimetals, contact materials) by using conventional techniques such as cold rolling, pressing, etc. is well known.

The preparation of amorphous bilayers by planar flow casting techniques has been reported previously [1]. However, the arrangement of melting two different alloys separately in two crucibles makes the technology of simultaneous casting of the two layers rather complicated and tedious. In our work we have used the technique of a single crucible with two nozzles close to each other and with a partition between them forming two separate vessels. Such an arrangement allows easy formation of two homogeneous layers along the whole ribbon length [2,3].

The aim of our work has been to investigate the formation and character of the bilayers, the connection of the two layers and the mutual intermixing of components at the interface between the layers. Magnetic properties of bilayers have been explored with respect to potential applications of such materials.

\section{Experimental}

Rapidly quenched bilayer ribbon with high quality surface and edges consisting of $\mathrm{Fe}_{77.5} \mathrm{Si}_{7.5} \mathrm{~B}_{15}$ (air side of the ribbon) and $\mathrm{Co}_{72.5} \mathrm{Si}_{12.5} \mathrm{~B}_{15}$ (wheel side) layers has been prepared by planar flow casting from a single crucible using the double-nozzle technique. Ribbons with typical thickness of $45-50 \mu \mathrm{m}(\approx$ twice the thickness of the usual single-layer ribbons) and $6 \mathrm{~mm}$ width exhibited amorphous structure of both layers according to $\mathrm{X}$-ray diffraction in as-quenched state, Fig. 1, identical to that of the single-layer ribbons with the respective compositions, which have been prepared as well via single-nozzle casting. Temperature dependences of electrical

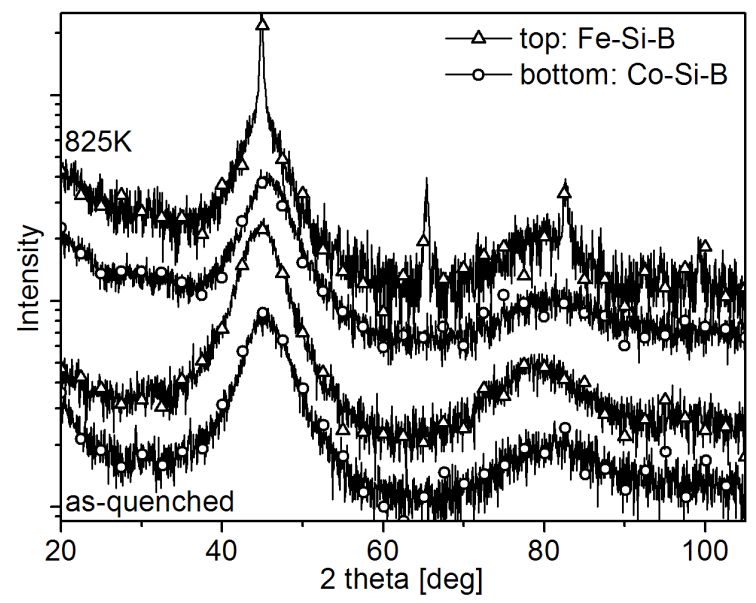

Fig. 1. XRD patterns from the top and bottom sides of the $\mathrm{Fe}-\mathrm{Si}-\mathrm{B} / \mathrm{Co}-\mathrm{Si}-\mathrm{B}$ bilayer sample in as-quenched state and after linear heating with $5 \mathrm{~K} / \mathrm{min}$ to $825 \mathrm{~K}$. The maxima on the $\mathrm{Fe}-\mathrm{Si}-\mathrm{B}$ layer after annealing corespond to bcc-Fe(Si).

resistivity $R(T)$, dilatation and "magnetic" weight (thermogravimetry, TGA, with weak permanent magnet) have 


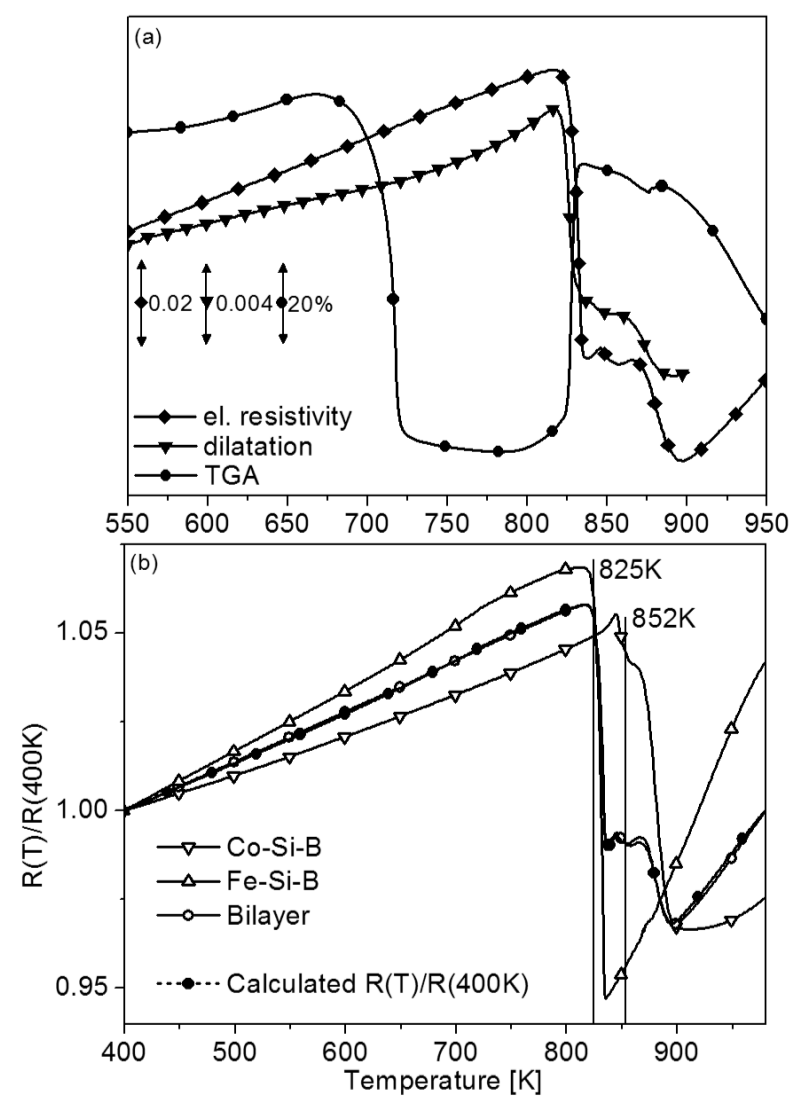

Fig. 2. (a) Temperature dependences of relative electrical resistivity $R(T) / R(400 \mathrm{~K})$, dilatation and TGA with permanent magnet; linear heating $5 \mathrm{~K} / \mathrm{min}$. (b) Relative electrical resistivities of the single layer $\mathrm{Fe}-\mathrm{Si}-\mathrm{B}$ and $\mathrm{Co}-\mathrm{Si}-\mathrm{B}$ samples and the calculated total electrical resistivity of the parallel combination of the two single layer samples compared to that of the measured bilayer ribbon.

been measured in amorphous state and during crystallization of both layers, which take place at different temperatures (Fig. 2a). The structure of the interlayer has been investigated by cross-sectional transmission electron microscopy (CS-TEM), X-ray diffraction (XRD, Cu $K_{\alpha}$ radiation) and scanning electron microscopy coupled to energy-dispersive X-ray spectroscopy (SEM/EDX).

\section{Results and discussion}

Temperature dependences of electrical resistivity and dilatation of bilayer ribbons in amorphous state (until $\approx 800 \mathrm{~K}$ ) exhibit the usual character of metallic glasses - low temperature coefficient of electrical resistivity (specific electrical resistivity of either of the layers is $\approx 130 \pm 10 \mu \Omega \mathrm{cm}$ ) and small positive thermal expansion coefficient. Crystallization starts above $800 \mathrm{~K}$ in the $\mathrm{Fe}-\mathrm{Si}-\mathrm{B}$ as witnessed by the decrease of electrical resistivity and contraction of the sample (corroborated by the $R(T)$ of single-layer ribbons in Fig. $2 \mathrm{~b}$ and XRD patterns taken at $825 \mathrm{~K}$ shown in Fig. 1). The Co-Si-B layer starts to crystallize above $\approx 850 \mathrm{~K}$. The remaining amorphous matrix in both layers transforms in the vicinity of $900 \mathrm{~K}$. TGA signal in weak magnetic field exhibits a decrease to nearly zero at $\approx 720 \mathrm{~K}$ due to the Curie temperature of the amorphous $\mathrm{Fe}-\mathrm{Si}-\mathrm{B}$ (the Curie temperature of amorphous $\mathrm{Co}-\mathrm{Si}-\mathrm{B}$ is $\approx 450 \mathrm{~K}$, not shown in the figure). The increase of the magnetic TGA signal correlates well with the formation of soft magnetic crystals of bcc-Fe( $\mathrm{Si})$. Its further increase above $850 \mathrm{~K}$ reflects the formation of Co-rich phases in the $\mathrm{Co}-\mathrm{Si}-\mathrm{B}$ layer.

Using the relative proportions of the measured inverse electrical resistivities of single layer $\mathrm{Fe}_{77.5} \mathrm{Si}_{7.5} \mathrm{~B}_{15}$ and $\mathrm{Co}_{72.5} \mathrm{Si}_{12.5} \mathrm{~B}_{15}$ ribbons as $0.48 / 0.52$, respectively, to compute the inverse electrical resistivity of the ideal parallel combination of the two layers yields the $R(T)$ dependence (calculated $R(T)$ curve in Fig. 2b) nearly identical to that of the original bilayer. This indicates, besides the expected nearly equal thickness of the two constituent layers of the bilayer, also only a minimal deviation from the superposition principle for electrical conductivities of parallel conductors. It suggests that the intermixing in the interface region is on very low level and does not change significantly even after complete crystallization of the layers upon linear heating. Similar effects and behavior have been observed by measurements of magnetic TGA and magnetization. The thickness of the individual layers $(23 \pm 2 \mu \mathrm{m}$ for the top Co-Si-B layer and $21 \pm 1 \mu \mathrm{m}$ for the bottom $\mathrm{Fe}-\mathrm{Si}-\mathrm{B}$ layer) has been estimated from SEM line profile analysis of Co and Fe, lower accuracy for the top layer resulting more from the air surface roughness than from the accuracy of the analysis itself.

Microscopical analysis of the bilayer has been performed on as-quenched samples as well as on samples heated to $825 \mathrm{~K}$ (Fig. 3) and $852 \mathrm{~K}$, which correspond to the temperatures after the onset of crystallization of $\mathrm{Fe}$ and Co based layers, respectively. Elemental mapping of Fe and Co performed by SEM/EDX analysis shows for all investigated samples a narrow well-defined interface of the layers the thickness of which does not exceed the resolution of method, which is $\approx 2 \mu \mathrm{m}$ for the chosen acquisition conditions. A more accurate analysis of constituent element maps by means of multivariate distribution analysis ([4] and references therein) indicates that this interface may be significantly narrower (Fig. 3c).

Higher magnification view of the interface has been obtained by CS-TEM observations (Fig. 4 - sample annealed to $825 \mathrm{~K}$ ), showing the separation between the entirely amorphous structure of Co based layer and partially crystallized structure of Fe based layer. It should be noted that no interface between Co based layer and remaining amorphous matrix in the Fe based layer can be observed which hints towards ideal interconnection of the original amorphous structures of the individual layers. The same sharp interface has been observed on the sample annealed to $852 \mathrm{~K}$ (not shown), separating the regions of growing crystals of different morphologies for 


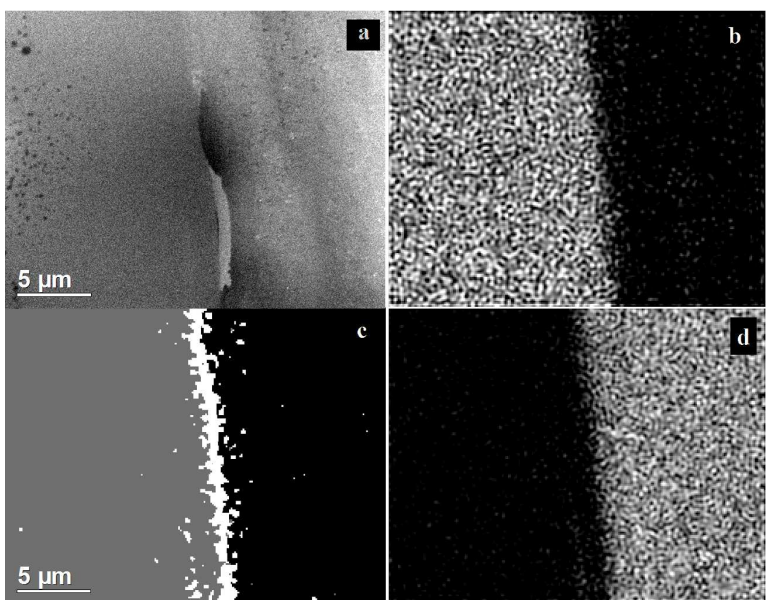

Fig. 3. (a) SEM image of the interface region after linear heating with $5 \mathrm{~K} / \mathrm{min}$ to $825 \mathrm{~K}$; (b), (d) elemental maps of Co and Fe from (a), respectively, taken at the same magnification; (c) composite bivariate Fe and Co map of the region shown in (a). The black and gray regions correspond to regions containing only $\mathrm{Fe}$ and only Co, respectively; the white area indicates the region positionally corresponding to the interface, where a non-zero mixing and simultaneous presence of both $\mathrm{Fe}$ and Co can be detected.

individual layers. These morphologies correspond well to those observed during the analogical analysis of crystallization of single amorphous layers of the corresponding stochiometries [5]. Possible nucleation and growth of intermetallic crystals in the interface region during higher-temperature transformation still needs to be verified.

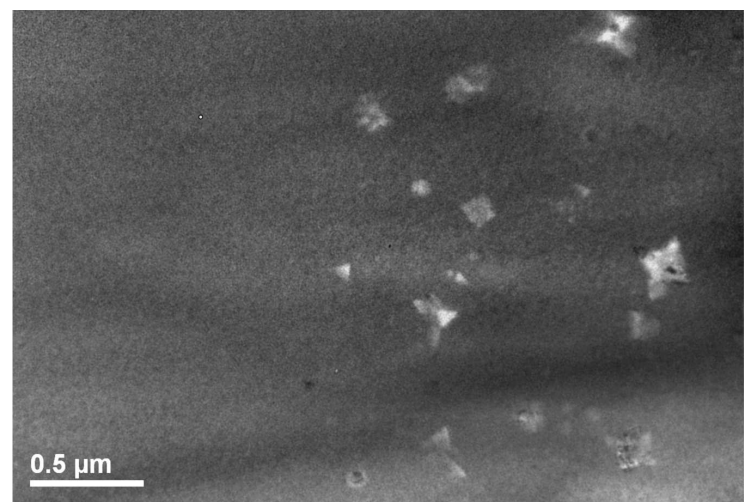

Fig. 4. CS-TEM image of the sample from Fig. 3 showing the interface between the still amorphous $\mathrm{Co}-\mathrm{Si}-\mathrm{B}$ layer (left) and partially crystallized $\mathrm{Fe}-\mathrm{Si}-\mathrm{B}$ layer (right).

It is worthwhile to note that while both layers are soft magnetic in amorphous and in crystallized states, additional magnetic effects may be expected due to the differences in thermal expansion coefficients of the two alloys ( Fe-Si-B in amorphous state exhibits a certain degree of invar effect below its Curie temperature) and due to the significant contraction of $\mathrm{Fe}-\mathrm{Si}-\mathrm{B}$ upon primary crystallization, imposing compressive stresses on the $\mathrm{Co}-\mathrm{Si}-\mathrm{B}$ layer which, in turn, stresses its $\mathrm{Fe}-\mathrm{Si}-\mathrm{B}$ counterpart. Suitably chosen annealing conditions may enhance these effects and provide means for their exploitation as unique magnetoelastic materials.

\section{Conclusions}

It was shown that bilayer ribbons with suitable chemical compositions of the two layers can be prepared in fully amorphous state by rapid quenching using double-nozzle technique. From the results it seems evident that the process of connection of the two layers during preparation takes place by solidification with only a small extent of mutual interdiffusion of component atoms localized to a narrow well-defined and nearly planar interface, leading to mechanically solid connection between the two layers. This is also in accord with the observed temperature dependences of electrical resistivity and magnetic thermogravimetry of the bilayer ribbons compared to those of single layer ribbons having the respective compositions of the top and bottom layers, which indicate only a minimal degree of deviation from the superposition principle.

Bilayers due to this character of the interface are suitable objects for further studies of the phenomena at the interfaces between two amorphous systems or between amorphous and crystalline phases, as well as of magnetoelastic phenomena due to differences in magnetic and mechanical character of the layers (in progress). Such materials yield readily the possibilities of their use as magnetoelastic sensors or magnetic actuators.

\section{Acknowledgments}

The work has been supported by the projects VEGA 2/0157/08, APVV-0413-06, APVV-0102-07 and by the CEX "Nanosmart".

\section{References}

[1] K. Inomata, S. Shimanuki, H. Yoshino, Bull. Jpn. Inst. Metals 20, 855 (1981).

[2] P. Duhaj, P. Švec, E. Majková, V. Boháč, I. Matłko, Matter. Sci. Eng. A 133, 662 (1991).

[3] T. Sato, Y. Sato, M. Fujikura, Y. Haga, J. Magn. Magn. Mater. 157/158, 211 (1996).

[4] P. Švec, J. Hoško, P. Švec, Sr., I. Mat'ko, P. Šebo, D. Janičkovič, in: Proc. 16th Int. Conf. on Applied Physics of Condensed Matter, APCOM 2010, Eds. J. Vajda, M. Weis, Slovak University of Technology, Bratislava 2010, p. 292.

[5] I. Mat'ko, E. Illeková, P. Švec, P. Duhaj, Mater. Sci. Eng. A 225, 145 (1997). 